\title{
The effect of suppressive oral acyclovir on the psychological morbidity associated with recurrent genital herpes
}

\author{
Orla Carney, Emma Ross, George Ikkos, Adrian Mindel
}

\begin{abstract}
Objectives-To assess the psychological impact of recurrent genital herpes and to determine if longterm acyclovir has any impact on this morbidity.
\end{abstract}

Setting and subjects-Patients with frequently recurring genital herpes attending a department of genitourinary medicine who were considered suitable for longterm acyclovir.

Methods-Patients completed an 80 item, self-administered psychological questionnaire before starting acyclovir and every three months for one year. Treatment was then stopped and three months later a further questionnaire was completed. The questionnaire consisted of the General Health Questionnaire (GHQ); the Hospital Anxiety and Depression Questionnaire (HADQ); Illness Attitude Scales and Illness Concern. Data were analysed by McNemar's test for changes in proportions and by Wilcoxon's test for changes in scores.

Results-102 patients were recruited: 55 men, and 47 women. Eighty two (80\%) patients completed three months treatment, 75 (74\%) six months, 64 (63\%) nine months and $61(60 \%)$ a year. Fifty (49\%) of the original 102 patients completed the three months post treatment follow up. At first visit $63 \%(64 / 102)$ were designated as GHQ "cases". Within three months this decreased to $26 \%$ (21/82). McNemar's test showed that $67 \%(34 / 51)$ of the patients who were initially classified as GHQ "cases" became "noncases" after three months $(p<0.0001)$. There was a significant decrease in the proportion of HAD anxiety cases from visit one to visit two ( $p<0.0001)$ and a decrease in illness concern scores from visit one to visit two (p< 0.0001). All these decreases were maintained throughout the years treatment with acyclovir.

Conclusions-There is a substantial morbidity associated with frequently recurring genital herpes. However, acyclovir suppression significantly reduces illness concern and anxiety and is a useful addition to the treatment of this infection.

(Genitourin Med 1993;69:457-459)

\section{Introduction}

Frequently recurring genital herpes infection can, depending on the severity of the recur- rences, be physically debilitating. The frequency and duration of such episodes can also place restrictions on the sexual behaviour and sexual activities of the individual. However, previous studies ${ }^{1-4}$ have suggested that there is a psychological morbidity associated with recurrent infection which is not necessarily attributable to the physical restrictions that frequently recurring infection may impose.

Although it has been hypothesised that stress is a factor in triggering recurrences, at least one study found no evidence for an increase in normal daily stress occurring prior to a recurrent episode of herpes. ${ }^{3}$ This is emphasised in Vanderplate and Aral's overview of published research. ${ }^{4}$ They suggest that "the subjective nature of such reports and the highly selective psychopathological nature of participants studied makes generalisation difficult".

One must accept that herpes does not exist in a vacuum. The media hysteria which greeted herpes in the early eighties has all but vanished in the era of HIV. ${ }^{5}$ The effects of this do still linger, however, and one needs to recognise the difficulties that many patients continue to have regarding sex and sexuality. ${ }^{6}$

With regard to genital herpes, one is faced with a recurrent, sexually transmitted, but dermatologically manifested infection. Populations studied with chronic illness, skin disorders, and sexually transmitted infections have been shown to have varying degrees of emotional dysfunctions, ${ }^{7-9}$ so that it would not be surprising to find that there is a psychological morbidity associated with recurrent genital herpes.

One of the most important areas of the body with regard to self image is the genitals, and those who have genital skin disorders may be manifestly depressed. ${ }^{10}$ Interestingly in their study on psychological approaches to the treatment of skin diseases, Beaman and Luzzato echo many of the themes which recur in writings about herpes. ${ }^{11}$ Shame, anxiety and isolation are all mentioned. Beaman and Luzzato come to the same conclusions as Aral and Vanderplate ${ }^{12}$ and Longo, Clum and Yaeger ${ }^{13}$ in their papers on the treatment and adjustment of patients to herpes. Problem solving skills based regimens coupled with good social support seem to bring maximum benefit to those affected by the common skin disorders such as psoriasis, as well as to those with genital herpes.

With the advent of the antiviral drug acyclovir we now have an effective treatment for genital herpes. Recent clinical studies have shown oral acyclovir to be highly successful in 
suppressing frequently recurring infection. ${ }^{14-16}$ Used continuously over periods of a year or more, acyclovir suppresses the virus, resulting in a substantial reduction in the frequency and severity of outbreaks; so that patients either experience no recurrences at all during the period of treatment, or at most only a few minor episodes.

We therefore conducted a study to assess: (1) The psychological morbidity of patients attending with frequently occurring genital herpes infection. (2) The impact of continuous oral acyclovir on this morbidity.

\section{Methods}

All patients with recurrent genital herpes who attended the Department of Genitourinary Medicine at the Middlesex Hospital between November 1987 and January 1991 and were eligible for continuous oral acyclovir were asked to participate in the study. Inclusion criteria included recurrent genital herpes for at least one year and at least eight recurrences per year. All patients had their diagnosis reconfirmed by viral culture. A control group was not included as it was considered to be unethical to withhold acyclovir in this group of patients.

Patients were asked to complete an 80 item, self-administered questionnaire, before starting treatment and every three months whilst on the treatment (which continued for 1 year) and once more three months after completing the year's course.

The questionnaire itself was divided into four sections, each being a well established psychological inventory. (1) General Health Questionnaire (GHQ). (2) Hospital Anxiety and Depression (HAD). (3) Illness Attitude Scales (IAS). (4) Illness Concern.

The GHQ measures non psychotic psychiatric illness, and responses are only counted as being present if the patient considers that they deviate from the norm. ${ }^{17}$ The 30 item inventory was used and using the standard convention "casesness" was taken as a score of 5 or more. One of the criticisms levelled against GHQ is that some of the responses may be influenced by concurrent physical illness. We therefore also used the HAD, since it avoids responses which may have a somatic

Table 1 Numbers of GHQ "cases" at each visit

\begin{tabular}{lllllll}
\hline Visit & 1 & 2 & 3 & 4 & 5 & 6 \\
\hline No of patients & & & & & & \\
$\quad$ at each visit & 102 & 82 & 75 & 64 & 61 & 50 \\
"Case" \% (No.) & $63(64)$ & $26(21)$ & $23(17)$ & $16(10)$ & $34(21)$ & $34(17)$ \\
"Noncase"\% (No.) & $37(38)$ & $74(61)$ & $77(58)$ & $84(54)$ & $66(40)$ & $66(33)$ \\
\hline
\end{tabular}

Table 2 Number of anxiety "cases" at each visit

\begin{tabular}{lllllll}
\hline Visit & 1 & 2 & 3 & 4 & 5 & 6 \\
\hline No. of patients & & & & & & \\
$\quad$ at each visit & 102 & 82 & 75 & 64 & 61 & 50 \\
Cases \% (No.) & $60(61)$ & $35(29)$ & $39(29)$ & $39(25)$ & $28(46)$ & $36(18)$ \\
Noncase \% (No.) & $40(41)$ & $65(53)$ & $61(46)$ & $61(39)$ & $54(33)$ & $64(32)$ \\
\hline
\end{tabular}

cause. ${ }^{18}$ Case definition using the standard convention is taken as a score of 8 or more in either of the anxiety or depression subscales.

The IAS measure fears, beliefs and attitudes associated with generalised abnormal illness behaviour. ${ }^{19}$ Conversely, Illness Concern assesses the actual and potential emotional impact caused by the specific problem which has brought them to the clinic, that is, frequently recurring genital herpes.

Data were analysed by McNemar's test for changes in proportions and by Wilcoxon's test for changes in scores. These tests were applied up to visit three where the response rate was above $70 \%$. To identify any changes over the study period, the percentages classified as cases at each visit have been presented in tables 1 and 2.

\section{Results}

102 patients were recruited: 55 men, and 47 women. There were no age differences between men and women (mean 32.0 vs $30 \cdot 8)$. Forty eight $(87 \%)$ of the men were heterosexual and seven homosexual. The majority of the patients (95\%) were employed. Most (62\%) were single. Eighty two $(80 \%)$ patients completed three months treatment, 75 (74\%) six months, 64 (63\%) nine months and $61(60 \%)$ a year. Fifty $(49 \%)$ of the original 102 patients completed the three month post treatment follow up. Although the numbers of participants decreases over time there was still a $60 \%$ response rate by the end of the year.

\section{General Health Questionnaire}

Table 1 shows the actual numbers of "cases" and "noncases". At first visit 63\% (64/102) were designated as "cases". Within three months this decreased to $26 \%(21 / 82)$, and this fall was maintained throughout their time on treatment, with a slight rise again as patients have either stopped or are about to finish, their year's treatment. McNemar's test showed that $67 \%(34 / 51)$ of the patients who were initially classified as GHQ "cases" became "noncases" after three months ( $p<$ $0.0001)$. There was no significant change from visit two to visit three.

Hospital Anxiety and Depression

There was a high level of acute anxiety amongst this population, with $60 \%(61 / 102)$ defined as "cases" before acyclovir was commenced. By the second visit this had almost halved to $35 \%(29 / 82)$, table 2. Using McNemar's test to assess any change in proportion from visit one to visit two, $43 \%$ (21/49) patients classified as anxiety "cases" before commencing treatment had become "noncases" within three months of commencing continuous acyclovir $(p<0.0001)$. Again, there was no change from visit two to visit three.

Acute depression was uncommon, with a negligibly small number of "cases" throughout the whole study: $14 \%(14 / 102)$ pretreatment, and $14 \%(7 / 50)$ post-treatment. 


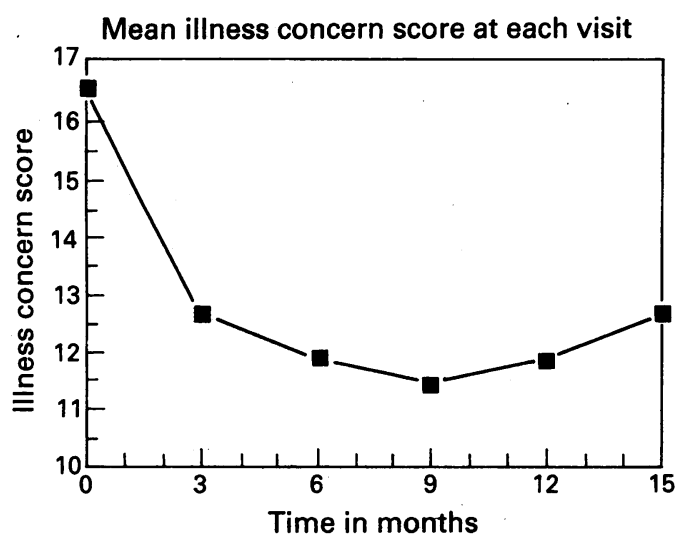

Figure Mean Illness Score for patients with frequently recurring genital herpes, showing a statistically significant decrease in scores between visit 1 (pretreatment) and visit 2 (after 3 months acyclovir suppression ( $p<0.0001)$ ).

\section{Illness Attitude Scales}

There were no significant changes observed either between groups or across time. There were only $13 / 102$ (13\%) "cases" of hypochondria at the onset of the study, and 4/50 $(8 \%)$ at the end of the study.

\section{Illness Concern (figure)}

As can be seen from the figure, when they first present patients have high scores. However, by the second visit, when they have had three months continuous treatment there is a dramatic decrease in overall scores, with $75 \%(54 / 72)$ of the patients scoring lower at visit two than at visit one $(p<0.0001) .{ }^{20}$ This remains fairly constant until the final visit, three months after stopping treatment, when there is a slight rise again, but still substantially below pretreatment level. There was no significant change in the scores from visit two to visit three.

\section{Discussion}

The study confirms that there is a substantial psychiatric morbidity in patients with frequently recurring genital herpes. At first visit, before treatment, many patients were greatly concerned about their infection and showed high levels of anxiety and depression. Yet within three months of commencing continuous acyclovir the percentage of GHQ cases had fallen from $63 \%$ to $26 \%$. In this initial three month period substantial decreases were also found in illness concern; and hospital anxiety. Between subsequent visits there were no significant differences suggesting that any attitude change occurred within three months of commencing continuous acyclovir.

One could surmise that the improvement in their wellbeing stems not from treatment, but from personal intervention and interest shown by the clinicians. However, many of the patients had been attending the clinic for a considerable period before attending the study, so that there was already a considerable patient/clinician relationship before treatment commenced.
From our work, we would suggest that it is the absence of the physical manifestations of a persistent, chronic sexually restricting, often painful disorder which improves mood, and not as previous work has suggested, that the patients' emotional state causes recurrences. There may also be a positive feedback with fewer recurrences in turn leading to an improved emotional state which in turn leads to fewer recurrences. Studies to compare the benefit of psychological intervention with continuos acyclovir would help to unravel this complex issue.

Continuous oral acyclovir will thus offer enormous benefit to those who experience emotional dysfunction because of their recurrences. Nevertheless acyclovir is not an antidote to good health education ${ }^{21}$ and should never be prescribed for emotional reasons alone. It is primarily treatment for those patients who have frequent recurrences and the improvement in psychological wellbeing should be considered as an extremely useful additional benefit.

1 Manne S, Sandler I. Coping and adjustment to Genital Herpes. $\mathcal{F}$ Behav Med 1984;7:4

2 Goldmeier D, Johnson A, Byrne M, Barton $S$. Psychosocial implications of recurrent genital herpes simplex virus infection. Genitourin Med 1988;64:327-30.

3 Rand K, Hoon E, Massey J, Johnson J. Daily stress and recurrence of genital herpes simplex. Arch Intern Med 1990;150:1889-93.

4 Vanderplate C, Aral S. Review: Psychological Aspects of Genital Herpes Virus Infection. Health Psychology 1987; 6:57-72.

5 Adler M, Mindel A. Genital Herpes: hype or hope? BMF 1983;286:1767-8.

6 Chenitz WC, Swanson JM. Counselling clients with genital herpes. Fournal of Psychosomatic Nursing and Mental Health Services 1989;27:11-7.

7 Pedder JR, Goldberg DP. A survey by questionnaire of psychiatric disturbance in patients attending a VD psychiatric disturbance in patients at
clinic. Brit $f$ Venereal Dis 1970;46:58-61.

8 Catalan J, Bradley M, Gallwey J, Hawton K. Sexual dysfunction and psychiatric morbidity in patients attending a clinic for sexually transmitted diseases. $\mathrm{Br} \mathcal{F}$ Psychiatry a clinic for sexually

9 Wessely SC, Lewis GH. The classification of psychiatric morbidity in attenders at a dermatology clinic. $B r f$ Psychiatry 1989;155:686-91.

10 Cotterill JA. Psychiatry and the skin. Br f Hosp Med 1989; 42:401-4.

11 Beaman K, Luzzato P. Psychological approaches to the treatment of skin diseases. Nursing 1988;29:1061-3.

12 Aral S, Vanderplate L, Magder L. Recurrent genital herpes: what helps adjustment? Sexually Transmitted Diseases 1988;15:164-6.

13 Longo D, Clum G, Yaeger N. Psychosocial treatment for recurrent genital herpes. fournal of Consulting and Clinical Psychology 1988;56:67-72.

14 Mindel A, Weller IVD, Faherty A, et al. Prophylactic oral acyclovir in recurrent genital herpes. Lancet 1988;11: $57-9$.

15 Kinghorn GR, Jeavons $M$, Rowland $M$, et al. Acyclovir prophylaxis of recurrent genital herpes: a randomised placebo controlled crossover study. Genitourin Med 1988;61:387-90.

16 Mindel A, Faherty A, Carney O, Patou G, Freris M Williams $P$. Dosage and safety of long term suppressive acyclovir therapy for recurrent genital herpes. Lancet
1988;1:926-8.

17 Goldberg DP. The detection of psychiatric illness by questionnaire. Maudsley Monographs, No 21 OUP. 1972

18 Barczak P, Kane N, Andrews S, Congdon AM, Clay JC, Betts T. Patterns of psychiatric morbidity in a genitoBetts T. Patterns of psychiatric morbidity in a
urinary clinic. Br $\mathcal{F}$ Psychiatry 1988;152:698-700.

19 Kellner R. Abridged Manual of the Ilness Attitude Scales. New Mexico: Department Psychiatry. School of

20 Siegel S. Non-parametric Statistics for the Behavioural Sciences. New York: McGraw-Hill. 1956 .

21 Swanson JM, Chenitz WC. Psychological aspects of genital herpes: a review of the literature. Public Health Nursing 1990;7:96-104. 